\title{
A Política Nacional de Alimentação e Nutrição e seu diálogo com a Política Nacional de Segurança Alimentar e Nutricional
}

\author{
The national food and nutrition policy \\ and its dialogue with the national food and nutrition security policy
}

Kelly Poliany de Souza Alves ${ }^{1}$

Patricia Constante Jaime ${ }^{1}$

${ }^{1}$ Coordenação Geral de

Alimentação e Nutrição,

Departamento de Atenção

Básica, Secretaria de

Atenção à Saúde, Ministério

da Saúde. SAF Sul - Quadra

2 - Lotes 5/6, Edifício

Premium - Bloco II -

Subsolo sala 8, Zona Cívico-

Administrativa. 70070-600

Brasília DF Brasil.

kelly.alves2@yahoo.com.br

\begin{abstract}
Food is one of the determinants and conditions of health and an inherent right of all people. The consequences of food and nutrition insecurity in the population, such as obesity, malnutrition and specific nutritional deficiencies, impact the health sector and have historically meant that it has assumed the responsibility for food and nutrition programs and policies in Brazil. However, ensuring food and nutrition security requires a combination of public policies, among which the National Food and Nutrition Policy of the Unified Health System (SUS) plays a fundamental role. This paper seeks to contribute to the debate on intersectoriality and health promotion based on presenting the National Food and Nutrition Policy and discussing its role as interface between the SUS and the National Food and Nutrition Security Policy and System. This perspective strongly suggests the combination of efforts to promote health and food and nutrition security in order to optimize initiatives developed in different sectors and accompanied by different policy councils that are not interrelated, enabling enhanced government and civil society action on the determinants of health and nutrition.
\end{abstract}

Key words Food security, Health promotion, Nutrition programs and policies
Resumo A alimentação é um dos determinantes e condicionantes da saúde e um direito inerente a todas as pessoas. As consequências da insegurança alimentar e nutricional da população, a exemplo da obesidade, desnutrição e carências nutricionais específicas, recaem sobre o setor saúde e têm feito com que, historicamente, este tenha incorporado a responsabilidade de politicas e programas de alimentação e nutrição no Brasil. Porém, a garantia da Segurança Alimentar e Nutricional exige uma conjunção de políticas públicas, dentre as quais a Política Nacional de Alimentação e Nutrição do SUS tem papel fundamental. O artigo objetiva contribuir com o debate sobre intersetorialidade e promoção da saúde a partir da apresentação da Política Nacional de Alimentação e Nutrição e discussão do seu papel enquanto interlocutora entre o Sistema Único de Saúde e a Politica e Sistema Nacional de Segurança Alimentar e Nutricional. Essa perspectiva sugere fortemente a conjunção de esforços para a promoção da saúde e de SAN, com vistas a potencializar agendas desenvolvidas em diferentes setores e acompanhadas por diferentes conselhos de politicas públicas, que não se articulam, permitindo uma melhor atuação do governo e da sociedade civil sobre os determinantes da saúde e da alimentação.

Palavras-chave Segurança alimentar e nutricional, Promoção da saúde, Programas e políticas de nutrição e alimentação 


\section{Introdução}

A promoção da saúde vai muito além de escolhas e práticas individuais e não está relacionada somente às responsabilidades do setor saúde, passa necessariamente pela garantia de condições sociais e econômicas que criem uma base favorável à adoção de estilos de vida saudável. Nesse sentido, a Organização Mundial da Saúde tem ressaltado a necessidade de que os países adotem a abordagem de Saúde em Todas as Políticas, na qual estabeleçam uma articulação entre todos os seus setores, para ampliar o desenvolvimento humano, a sustentabilidade e a equidade, assim como melhorar as condições de saúde ${ }^{1,2}$. No Brasil, a Política Nacional de Promoção da Saúde (PNPS) reconhece a impossibilidade do setor saúde de responder sozinho à transformação dos determinantes e condicionantes para garantir opções saudáveis para a população e aponta o desafio da sua participação na construção de estratégias e ações intersetoriais que propiciem uma articulação das responsabilidades dos distintos setores sobre a complexa determinação da saúde ${ }^{3}$.

A alimentação é um dos determinantes e condicionantes da saúde e um direito inerente a todas as pessoas. A promoção e garantia de uma alimentação adequada e saudável tem mobilizado historicamente esforços de diferentes setores do governo brasileiro e também de entidades e movimentos da sociedade civil, mas que nem sempre apresentaram os mesmos objetivos ou foram complementares. Programas de distribuição de alimentos, por exemplo, estiveram por vezes desconectados de estratégias estruturantes de melhoria das condições socioeconômicas da população, para que esta obtivesse os meios necessários para acessar os alimentos de forma autônoma ${ }^{4-9}$.

Com grande envolvimento da sociedade civil organizada e uma abertura na agenda política no âmbito do governo federal, o Brasil vem consolidando na última década a gestão intersetorial de políticas públicas, com o objetivo comum de promover a Segurança Alimentar e Nutricional - SAN, compreendida como a realização do direito de todos ao acesso regular e permanente a alimentos de qualidade, em quantidade suficiente, sem comprometer o acesso a outras necessidades essenciais, tendo como base práticas alimentares promotoras de saúde que respeitem a diversidade cultural e que sejam ambiental, cultural, econômica e socialmente sustentáveis ${ }^{10}$.

Este artigo objetiva contribuir com o debate sobre intersetorialidade e promoção da saúde a partir da apresentação da Política Nacional de
Alimentação e Nutrição (PNAN) e discussão do seu papel enquanto interlocutora entre o Sistema Único de Saúde (SUS) e a Política e Sistema Nacional de Segurança Alimentar e Nutricional (SISAN). Está organizado em quatro sessões, além desta introdução: a primeira trata sobre a interdependência entre os direitos à saúde e alimentação; a segunda sessão aborda o diálogo entre a Política Nacional de Alimentação e Nutrição (PNAN) e a Política Nacional de Segurança Alimentar e Nutricional (PNSAN), apresentando as agendas de alimentação e nutrição e de outras áreas do SUS, que compõe as prioridades do I Plano Nacional de SAN; a terceira sessão aborda os mecanismos de governança do SISAN no âmbito do Governo Federal, que permitem a participação do SUS em sua gestão intersetorial; e a última sessão apresenta as considerações acerca dos desafios da gestão intersetorial.

\section{Saúde e alimentação: indissociabilidade de direitos e determinantes}

Os direitos à saúde e à alimentação são inalienáveis e independem de legislação nacional, estadual ou municipal específica, pois tratam de direitos humanos os quais todos possuem, única e exclusivamente, por terem nascido e serem humanos. No entanto, o reconhecimento desses direitos em normas, acordos, declarações e em outros instrumentos se torna importante para assegurar seu cumprimento pelo Estado e sua exigibilidade pelos seus titulares. A Constituição Brasileira, promulgada em 1988, reconheceu a saúde como um direito social, mas o direito à alimentação só foi incluído entre estes no ano de 2010, por meio da Emenda Constitucional No $64^{11}$.

Os direitos humanos são indivisíveis, interdependentes e inter-relacionados em sua realização. Isso significa que todos os direitos são igualmente necessários para uma vida digna, que a satisfação de um não pode ser usada como justificativa para a não realização de outros. A garantia da SAN exige que o direito à saúde seja respeitado e concretizado para que seja alcançado seu fim, que é a realização da alimentação e nutrição adequados. Da mesma forma, a garantia da saúde também depende de ações de diversas áreas que garantam a realização dos demais direitos humanos, entre eles o direito à alimentação adequada, que constituem a base para a plena saúde em seu conceito mais amplo. Ou seja, para se ter saúde é preciso ter SAN, para se ter SAN é preciso ter saúde ${ }^{12-14}$.

A perspectiva dos determinantes sociais de saúde (DSS), entendidos como os fatores so- 
ciais, econômicos, culturais, étnicos/raciais, psicológicos e comportamentais, que influenciam a ocorrência de problemas de saúde e seus fatores de risco na população, contribui para a compreensão da inter-relação entre saúde e SAN ${ }^{15}$. Nesse sentido, pode-se compreender que a promoção da saúde e da Segurança Alimentar e Nutricional, também são interdependentes e inter-relacionadas. Como aponta Pinheiro ${ }^{16}$, a promoção da alimentação adequada e saudável é uma zona de intersecção entre a promoção da saúde e a promoção de SAN, podendo aproximar e subsidiar o diálogo intersetorial, imprescindível tanto para a garantia da SAN quanto da saúde, pois nesta análise, os fatores determinantes da saúde também vão influenciar na condição de SAN dos indivíduos e coletividades. E o conceito abrangente de saúde, que não se restringe somente na capacidade física ou condição biológica dos sujeitos, individualmente, se concretiza mediante a garantia da SAN.

Essa perspectiva sugere fortemente a conjunção de esforços para a promoção de saúde e de SAN, o que viria a potencializar agendas desenvolvidas em diferentes setores e acompanhadas por diferentes conselhos de políticas públicas que não se articulam. O que permitiria maior e melhor atuação do governo e da sociedade civil sobre os determinantes da saúde e da alimentação.

\section{Diálogo entre a Política Nacional de Alimentação e Nutrição e a Política Nacional de Segurança Alimentar e Nutricional}

As consequências da insegurança alimentar e nutricional da população, a exemplo da desnutrição e carências nutricionais específicas, recaem sobre o setor saúde e tem feito com que historicamente este setor tenha incorporado a responsabilidade de políticas e programas de alimentação e nutrição no Brasil. No entanto, a garantia da Segurança Alimentar e Nutricional exige uma conjunção de políticas públicas, dentre as quais a Política Nacional de Alimentação e Nutrição do SUS tem papel fundamental ${ }^{17}$.

No final da década de 1990, em meio ao enfraquecimento do tema da SAN na agenda pública nacional, foi formulada a Política Nacional de Alimentação e Nutrição ${ }^{18}$ (PNAN), a partir da luta e contribuições de atores de instituições governamentais e não governamentais com atuação no campo da alimentação e nutrição. A homologação dessa política foi considerada um meio para garantir dentro do governo um espaço para a SAN, uma vez que o Conselho Nacional de Se- gurança Alimentar e Nutricional (CONSEA) e o Instituto Nacional de Alimentação e Nutrição (INAN), autarquia vinculada ao Ministério da Saúde, haviam sido extintos. Desta forma, representa uma conquista no que se refere à legitimação das ações nesta área e na definição da contribuição do setor saúde para garantia da SAN e concretização do direito humano à alimentação, reafirmando a necessidade de diálogo e articulação para a realização de ações que não se restringem ao setor saúde, mas precisam estar em contínua interação com outros setores ${ }^{4,5,19}$.

A PNAN apontou que a atuação do setor saúde no contexto da SAN é marcada por dois momentos denominados positivo e crítico. O primeiro ocorre quando há garantia da SAN em todas as suas dimensões. Nesse caso, as ações predominantes do setor saúde são a vigilância alimentar e nutricional, a vigilância sanitária de alimentos e as medidas de caráter educativo. $\mathrm{O}$ momento crítico ocorre quando há falhas na garantia da SAN, seja na sua dimensão alimentar, prejudicando o acesso a alimentos em quantidade e qualidade adequadas, ou na sua dimensão nutricional, com agravos à saúde que prejudicam a utilização biológica dos alimentos e de práticas alimentares não saudáveis que podem desencadear carências específicas, obesidade e outras doenças. Nesse momento cabe ao setor saúde a oferta adequada dos serviços necessários ao tratamento e reabilitação, bem como a prevenção de novos agravos ${ }^{18}$.

Entre as ações a serem desenvolvidas pelo setor saúde no momento positivo, Valente et al. ${ }^{13}$ acrescentam a oferta de serviços de atenção básica à saúde, como: vacinação, exames preventivos, pré-natal, promoção do aleitamento materno exclusivo, acompanhamento do crescimento e desenvolvimento, tratamento de doenças mais comuns, etc. Por outro lado, apontam como obstáculos setoriais que contribuem para o momento crítico a baixa cobertura ou má qualidade dos serviços prestados à população.

A primeira versão da PNAN teve como propósito a garantia da qualidade dos alimentos colocados para consumo no País, a promoção de práticas alimentares saudáveis e a prevenção e o controle dos distúrbios nutricionais. Considerando que, para o alcance deste propósito, seria necessária a articulação intersetorial, foram descritos em seu texto algumas instituições e setores federais prioritários, bem como as principais medidas preconizadas para a articulação com cada um deles ${ }^{18}$. Assim, a PNAN insere na saúde o debate da SAN e traz para o contexto intersetorial a contribuição da saúde. 
Compreende-se que explicitar tais questões em uma política setorial foi estratégico para o conjunto de atores que construíram a PNAN diante da inexistência de instâncias ou normativas de governo naquele momento que promovessem tais articulações para a promoção da SAN.

Após dez anos de publicação da PNAN, Recine e Vasconcelos ${ }^{20}$ apontaram como méritos de sua implementação: o desenvolvimento da vigilância alimentar e nutricional (VAN), que permitiu a produção sistemática de informações sobre a situação alimentar e nutricional da população brasileira, a partir da implementação do Sistema de Vigilância Alimentar e Nutricional (SISVAN) e de inquéritos nacionais, como as Pesquisas de Orçamentos Familiares (POF) e a vigilância de fatores de risco e proteção para doenças crônicas por inquérito telefônico (Vigitel); a construção da agenda de promoção da alimentação adequada e saudável e sua qualificação a partir da edição do Guia Alimentar para a População Brasileira ${ }^{21}$, que se apresenta como uma ferramenta brasileira para implementação das recomendações da Estratégia Global de Promoção da Alimentação Saudável, Atividade Física e Saúde ${ }^{22}$; além da qualificação de recursos humanos em alimentação e nutrição, sobretudo a partir do trabalho da rede de Centros Colaboradores de Alimentação e Nutrição (CECAN), formada por instituições públicas nas cinco regiões brasileiras, buscando integrar ensino, pesquisa e serviço.

A conjunção das novas necessidades de saúde da população brasileira, derivadas de modificações no quadro epidemiológico e socioeconômico, com as inovações nos mecanismos de gestão e organização da atenção à saúde adotadas no SUS e as responsabilidades do setor saúde para promoção de SAN junto ao SISAN, nortearam o processo de revisão da PNAN, realizado entre os anos de 2010 e 2011. Este processo teve como desafio atualizar as diretrizes dessa política de forma a orientar a organização e qualificação das ações de alimentação e nutrição nas Redes de Atenção à Saúde ${ }^{23}$ (RAS) e também de legitimá-la como interlocutora entre o SUS e o SISAN ${ }^{24}$.

A Comissão Intersetorial de Alimentação e Nutrição do Conselho Nacional de Saúde (CIANCNS) e o Ministério da Saúde apontaram como desafios para a PNAN, no seu processo de revisão: qualificação da gestão das ações de alimentação e nutrição; fortalecimento das estratégias de implantação da nutrição na atenção básica e nos demais níveis de atenção à saúde; delineamento de ações destinadas a populações específicas (indígenas e outros povos e comuni- dades tradicionais); reconhecimento e valorização da cultura alimentar; ampliação da discussão relativa à temática de nutrição, ambiente, desenvolvimento e aproximação com as instâncias de controle social da saúde e da nutrição ${ }^{25}$.

A nova versão da PNAN ${ }^{26}$, aprovada em 2011, apresenta como propósito a melhoria das condições de alimentação, nutrição e saúde da população brasileira, mediante a promoção de práticas alimentares adequadas e saudáveis, a vigilância alimentar e nutricional, a prevenção e o cuidado integral dos agravos relacionados à alimentação $e$ nutrição. Tem por pressupostos os direitos à saúde e à alimentação e é orientada pelos princípios doutrinários e organizativos do SUS, aos quais se somam os princípios: alimentação como elemento de humanização das práticas de saúde; respeito à diversidade e à cultura alimentar; fortalecimento da autonomia dos indivíduos; determinação social e a natureza interdisciplinar e intersetorial da alimentação e nutrição; e Segurança Alimentar e Nutricional com soberania.

Para alcance de seu propósito, a PNAN traz um conjunto de diretrizes que norteiam a organização e oferta dos cuidados relativos à alimentação e nutrição no SUS, que devem contribuir para a conformação de uma rede integrada, resolutiva e humanizada de cuidados, sendo elas: Organização da Atenção Nutricional; Promoção da Alimentação Adequada e Saudável; Vigilância Alimentar e Nutricional; Gestão das Ações de Alimentação e Nutrição; Participação e Controle Social; Qualificação da Força de Trabalho; Controle e Regulação dos Alimentos; Pesquisa, Inovação e Conhecimento em Alimentação e Nutrição; Cooperação e articulação para a SAN ${ }^{26}$.

Com a instituição da SAN, enquanto política pública de Estado, a estruturação de um Plano Nacional e a organização de um Sistema intersetorial para sua implementação ${ }^{10,24}$, a nova versão da PNAN reafirma o compromisso de cooperação e articulação do SUS para essa política intersetorial, mas reforça a articulação intrasetorial, buscando apresentar de forma mais clara a articulação entre suas diretrizes e outras políticas e mecanismos de gestão do SUS. A diretriz específica que trata da articulação entre SUS e SISAN refere que esta proporcionará a articulação dos cuidados em alimentação e nutrição na RAS às demais ações de SAN nos territórios, com vistas ao enfrentamento da insegurança alimentar e nutricional e dos agravos em saúde, na ótica de seus determinantes sociais.

Nesse sentido, são destacadas na PNAN, entre outras, as ações direcionadas: (i) à melhoria 
da saúde e nutrição das famílias beneficiárias de programas de transferência de renda, implicando ampliação do acesso aos serviços de saúde; (ii) à interlocução com os setores responsáveis pela produção agrícola, distribuição, abastecimento e comércio local de alimentos, visando o aumento do acesso a alimentos saudáveis; (iii) à promoção da alimentação adequada e saudável em ambientes institucionais como escolas, creches, presídios, albergues, locais de trabalho, hospitais, restaurantes comunitários, entre outros ${ }^{26}$.

É comum relacionar as contribuições do SUS para a SAN apenas às ações coordenadas pela área técnica de alimentação e nutrição do Ministério da Saúde, sobretudo a vigilância alimentar e nutricional, as ações de promoção da alimentação adequada e saudável e os programas de prevenção de carências nutricionais. No entanto, partindo da compreensão da interdependência entre saúde e SAN, todo o conjunto de ofertas do SUS para promoção e proteção da saúde, vigilância em saúde, prevenção, diagnóstico, tratamento e reabilitação de agravos e doenças, contribuem para a SAN.

Avanços nessa compreensão se concretizaram no I Plano Nacional de Segurança Alimentar e Nutricional 2012-2015 ${ }^{27}$ (PLANSAN), publicado em 2011, onde foram incluídas metas e iniciativas desenvolvidas por diferentes áreas do Ministério da Saúde e unidades vinculadas. Além da Coordenação Geral de Alimentação e Nutrição do Departamento de Atenção Básica da Secretaria de Atenção à Saúde (CGANDABSAS), são responsáveis pela execução e monitoramento de metas do PLANSAN: Coordenação Geral de Saúde da Criança e Aleitamento Materno (CGSCAMDAPESSAS), Secretaria Especial de Saúde Indígena (SESAI), Fundação Nacional de Saúde (Funasa) e Agência Nacional de Vigilância Sanitária (Anvisa).

O PLANSAN explicita as responsabilidades dos órgãos e entidades da União para consolidação de programas e ações relacionadas às diretrizes da Política Nacional de SAN ${ }^{27}$, que são:

I - Promoção do acesso universal à alimentação adequada e saudável, com prioridade para as famílias e pessoas em situação de insegurança alimentar e nutricional;

II - Promoção do abastecimento e estruturação de sistemas sustentáveis e descentralizados, de base agroecológica, de produção, extração, processamento e distribuição de alimentos;

III - Instituição de processos permanentes de educação alimentar e nutricional, pesquisa e formação nas áreas de segurança alimentar e nutricional e do direito humano à alimentação adequada;

IV - Promoção, universalização e coordenação das ações de segurança alimentar e nutricional voltadas para quilombolas e demais povos e comunidades tradicionais de que Trata o Decreto $n^{\circ} 6.040 / 2007$, povos indígenas e assentados da reforma agrária;

V - Fortalecimento das ações de alimentação e nutrição em todos os níveis da atenção à saúde, de modo articulado às demais ações de segurança alimentar e nutricional;

VI - Promoção do acesso universal à água de qualidade e em quantidade suficiente, com prioridade para as famílias em situação de insegurança hídrica e para a produção de alimentos da agricultura familiar e da pesca e aquicultura;

VII - Apoio a iniciativas de promoção da soberania alimentar, segurança alimentar e nutricional e do direito humano à alimentação adequada em âmbito internacional e a negociações internacionais baseadas nos princípios e diretrizes da Lei no 11.346, de 2006; e

VIII - Monitoramento da realização do direito humano à alimentação adequada.

$\mathrm{Na}$ diretriz II foram incluídas metas de responsabilidade da Anvisa, sobre o aperfeiçoamento dos mecanismos de gestão, controle e educação voltados para o uso de agrotóxicos, organismos geneticamente modificados e demais insumos agrícolas. Dentre estes está o Programa de Análise de Resíduos de Agrotóxicos em Alimentos (PARA), que avalia a qualidade e a segurança dos alimentos consumidos pela população quanto ao nível de resíduos de agrotóxicos.

$\mathrm{Na}$ diretriz III consta como meta de responsabilidade do Ministério da Saúde a atualização do Guia Alimentar para a população brasileira e do Guia de Alimentos Regionais Brasileiros, ambos produzidos pela Coordenação Geral de Alimentação e Nutrição (CGAN).

As metas de responsabilidade da Funasa e Sesai foram incluídas na diretriz IV. Dizem respeito à ampliação da cobertura de ações e serviços de saneamento básico e serviços de abastecimento de água em comunidades quilombolas, assentamentos rurais, terras indígenas e demais territórios de povos e comunidades tradicionais, priorizando soluções alternativas que permitam a sustentabilidade dos serviços; e também à garantia da atenção integral à saúde das famílias indígenas a partir do subsistema de saúde indígena e sua articulação com as práticas e medicinas tradicionais, com participação popular e articulação intersetorial. 
Todas as metas estabelecidas na diretriz V são de responsabilidade do setor saúde e a Anvisa e a CGAN são as áreas técnicas responsáveis pela quase totalidade delas. As metas dessa diretriz visam atingir quatro objetivos: a) controlar e prevenir os agravos e doenças consequentes da insegurança alimentar e nutricional; b) promover o controle e a regulação de alimentos; c) estruturar a atenção nutricional na Rede de Atenção à Saúde; e d) fortalecer a vigilância alimentar e nutricional.

Compete à Anvisa as metas relacionadas à regulação da publicidade de alimentos ultraprocessados; ao monitoramento da iodação do sal comercializado para consumo humano e dos teores de sódio, açúcares e gorduras em alimentos processados; à elaboração de plano preparatório para a prevenção e o controle de agravos relacionados aos alimentos durante os eventos de massa com ênfase na Copa do Mundo de 2014 e nos Jogos Olímpicos de 2016; ao desenvolvimento de ações voltadas para grupos populacionais com necessidades alimentares especiais, incluindo a atualização e informatização da tabela de informação sobre o teor de fenilalanina nos alimentos para orientar a dieta dos portadores de fenilcetonúria, entre outras.

A CGAN é responsável pelas estratégias para cumprimento das metas relativas à prevenção de carências de ferro, vitamina A e vitamina B1 (beribéri); atualização da PNAN; garantia de equipamentos antropométricos para as Unidades Básicas de Saúde; aumento de cobertura do Sistema de Vigilância Alimentar e Nutricional (SISVAN); apoio a estudos e pesquisas que possam trazer subsídios para a análise do estado nutricional e do consumo alimentar e nutricional da população brasileira; elaboração de um Plano Nacional Intersetorial de prevenção e controle da obesidade; pactuação da redução do teor de sódio nos alimentos processados prioritários; expansão dos Centros Colaboradores de Alimentação e Nutrição e implementação da Rede Social de Nutrição do SUS (RedeNutri).

Ainda no escopo dessa diretriz, compete a CGSCAMDAPESSAS a meta referente à expansão dos bancos de leite humano. Cabe ressaltar que, para cumprimento das metas de sua responsabilidade, a CGAN necessita articular-se com outras coordenações do próprio Departamento de Atenção Básica e outras áreas do Ministério da Saúde, como o Departamento de Assistência Farmacêutica (DAFSCTIE) e o Departamento de Informática do SUS (DatasusSEGEP), além de outras instituições parceiras como a Organização
Pan-americana de Saúde (OPAS), universidades e o Instituto Brasileiro de Geografia e Estatística (IBGE), e, sobretudo, com as Secretarias Estaduais e Municipais de Saúde.

Embora cada ente federado possua autonomia política e financeira, para a garantia da atenção integral à saúde há a necessidade de responsabilização compartilhada, solidária e cooperativa entre o Ministério da Saúde e as Secretarias Estaduais, do Distrito Federal e Municipais de Saúde. Nesse sentido, a articulação interfederativa é pressuposto para a construção e implementação das políticas, programas e ações no âmbito do SUS, promovida pela discussão, negociação e pactuação das demandas apresentadas pelos entes federativos objetivando a melhor solução para as questões.

Muitas outras agendas do SUS, além das que foram incluídas como prioritárias no I PLANSAN, trazem contribuições importantes que corroboram para a promoção de SAN. Dentre elas, podemos citar:

- Todo o investimento para melhoria do acesso e da qualidade dos serviços que compõe as Redes de Atenção à Saúde (RAS), em especial no âmbito da Atenção Básica (AB), com ampliação da cobertura populacional, reforma e construção de unidades básicas, provimento de profissionais e implementação de equipes de apoio matricial (Núcleos de Apoio à Saúde da Família);

- O acompanhamento sistemático das condicionalidades de saúde dos beneficiários do Programa Bolsa Família (PBF) realizado pelas equipes de Atenção Básica de todo o país;

- O conjunto de ações desenvolvidas no escopo do Programa Saúde na Escola (PSE), pela parceria das equipes de $\mathrm{AB}$ com os profissionais de educação das escolas nos territórios;

- A Política Nacional de Promoção da Saúde, na qual a promoção da alimentação adequada e saudável é uma das agendas prioritárias ${ }^{3}$;

- O Plano de ações estratégicas para o enfrentamento das doenças crônicas não transmissíveis (DCNT) no Brasil 2011-2022, com definição de metas e ações do Ministério da Saúde a serem desenvolvidas em parceria com outros setores ${ }^{28}$. A publicação das diretrizes para a organização da prevenção e do tratamento do sobrepeso e obesidade como linha de cuidado prioritária da Rede de Atenção à Saúde das Pessoas com Doenças Crônicas ${ }^{29}$, e seus desdobramentos junto às Secretarias Estaduais e Municipais de Saúde;

- As políticas de promoção da equidade e seus respectivos planos operativos, construídas em parceria com a sociedade civil organizada e com 
os movimentos sociais, que objetivam diminuir as diferenças históricas no acesso ao SUS por parte de diversos grupos vulneráveis como as populações Negra, LGBT, do Campo, da Floresta e das Águas, Cigana e em Situação de Rua ${ }^{30}$;

- A Política Nacional de Educação Popular em Saúde (PNEP-SUS), que visa implementar a Educação Popular em Saúde no âmbito do SUS, compreendendo-a como prática político-pedagógica orientadora de processos educativos e de trabalho social emancipatório, buscando levar a autonomia às pessoas e a horizontalidade entre os saberes técnico-científicos e populares e considerando fundamental o respeito à diversidade, o enfrentamento das desigualdades sociais e de qualquer forma de discriminação e opressão ${ }^{31}$;

- Os avanços na discussão sobre atenção às necessidades alimentares especiais no SUS, com construção de orientações técnicas para a organização da terapia nutricional na RAS, de forma participativa junto a sociedades de especialistas, instituições de ensino e pesquisa, gestores e profissionais do SUS.

Entre os atuais desafios para ampliar as contribuições do SUS para a SAN estão: a inclusão desta temática nos processos de formação e educação permanente dos profissionais de saúde, a exemplo dos cursos promovidos no escopo do Programa de Valorização dos Profissionais da Atenção Básica (PROVAB) e do Telessaúde Brasil Redes; e a implementação do Programa de Aquisição de Alimentos (PAA), junto a rede hospitalar do SUS, visando promover a doação e aquisição de produtos da agricultura familiar para a produção de refeições nesses serviços de saúde por meio da articulação junto ao Ministério do Desenvolvimento Social e Combate à fome (MDS) e Ministério do Desenvolvimento Agrário (MDA).

\section{Mecanismos de governança da Política Nacional de Segurança Alimentar e Nutricional no âmbito federal}

Um recente arcabouço legal institucionaliza os mecanismos de governança da Política Nacional de Segurança Alimentar e Nutricional (PNSAN). A Lei No 11.346 de 15 de setembro de 2006, Lei Orgânica de Segurança Alimentar e Nutricional (LOSAN), criou o SISAN, estabelecendo seus princípios, diretrizes, objetivos e composição, por meio do qual o poder público, com a participação da sociedade civil organizada, deverá formular e implementar políticas, planos, programas e ações com vistas em assegurar o direito humano à alimentação adequada ${ }^{10}$.
O SISAN tem por objetivos formular e implementar Políticas e Planos de Segurança Alimentar e Nutricional, estimular a integração dos esforços entre governo e sociedade civil, bem como promover o acompanhamento, o monitoramento e a avaliação da Segurança Alimentar e Nutricional do País ${ }^{10}$. Integram esse Sistema:

1. A Conferência Nacional de Segurança Alimentar e Nutricional (CNSAN), instância responsável pela indicação ao Conselho Nacional de SAN (CONSEA) das diretrizes e prioridades da Política e do Plano Nacional de SAN, bem como pela avaliação do SISAN;

2. O CONSEA, órgão de assessoramento imediato ao Presidente da República, a quem cabe, entre outras competências, articular, acompanhar e monitorar, em regime de colaboração com os demais integrantes do Sistema, a implementação e a convergência de ações inerentes à Política e ao Plano Nacional de SAN;

3. A Câmara Interministerial de Segurança Alimentar e Nutricional (CAISAN), integrada por Ministros de Estado e Secretários Especiais responsáveis pelas pastas afetas à consecução da SAN, com as atribuições de elaborar, a partir das diretrizes emanadas do CONSEA, a Política e o Plano Nacional de SAN, indicando diretrizes, metas, fontes de recursos e instrumentos de acompanhamento, monitoramento e avaliação de sua implementação;

4. Os órgãos e entidades de SAN da União, dos Estados, do Distrito Federal e dos Municípios; e,

5. As instituições privadas, com ou sem fins lucrativos, que manifestem interesse na adesão e que respeitem os critérios, princípios e diretrizes do SISAN.

À exemplo do SUS, o SISAN também prevê a participação e o controle social por meio do CONSEA e conferências de SAN. Mas, o CONSEA se constitui em um órgão de assessoramento do Presidente da República tendo caráter consultivo, diferente dos Conselhos de Saúde que tem caráter deliberativo para o SUS. É composto por dois terços de representantes da sociedade civil e um terço de representantes governamentais, sendo responsável por convocar e organizar as conferências de SAN e propor à CAISAN as diretrizes e prioridades do PLANSAN, incluindo-se os requisitos orçamentários para sua consecução ${ }^{32}$.

A CAISAN tem a finalidade de promover a articulação e a integração dos órgãos e entidades da administração pública federal afetos à área de SAN. Deve elaborar, a partir das diretrizes emanadas do CONSEA, a Política e o Plano Nacional 
de Segurança Alimentar e Nutricional, indicando neste último as metas, fontes de recursos e instrumentos de acompanhamento, monitoramento e avaliação de sua execução. O Plano Ministerial da CAISAN é composto pelos representantes governamentais dos Ministérios que são membros natos do CONSEA ${ }^{33}$. O Ministério da Saúde é membro do CONSEA e da CAISAN, sendo seu representante titular o Ministro da Saúde e suplente o Coordenador Geral de Alimentação e Nutrição.

Somente em 2010 foi publicado o Decreto que regulamenta a LOSAN, institui a Política Nacional de Segurança Alimentar e Nutricional (PNSAN) e estabelece os parâmetros para a elaboração do PLANSAN, sendo este o principal instrumento de planejamento, gestão e execução da Política Nacional de SAN ${ }^{26}$. O I PLANSAN tem a mesma vigência do Plano Plurianual - PPA (20122015) e está estreitamente relacionado às metas e iniciativas nele definidas, o que traz para a esfera estratégica do planejamento da ação pública a garantia das condições de sua implementação.

\section{Considerações finais}

A incorporação da intersetorialidade nas políticas públicas voltadas a promoção da Segurança Alimentar e Nutricional tem possibilitado a articulação de diferentes áreas técnicas de governo que passaram a integrar agendas coletivas e compartilhar objetivos comuns. Nesta perspectiva, a intersetorialidade tem grande potencial para produzir melhores condições para garantia de direitos à população. Porém, novos problemas e desafios relacionados à superação da fragmenta- ção e à articulação das políticas públicas emergem nos espaços de negociação e construção compartilhada. Como a hierarquia de poder no âmbito das políticas públicas em que se destacam as políticas macroeconômicas.

Ainda entre os desafios, cabe destacar a necessidade de promover o diálogo e articulação também entre os diferentes conselhos de controle social das políticas públicas, a exemplo do CONSEA e do Conselho Nacional de Saúde (CNS), com vistas a ampliar o debate e participação dos diferentes segmentos da sociedade na definição de prioridades para políticas intersetoriais e no seu monitoramento. A Comissão Intersetorial de Alimentação e Nutrição (CIAN) do CNS precisa ser reconhecida e utilizada como um espaço para esse diálogo.

A efetiva intersetorialidade necessita que a articulação dos diferentes setores ocorra em todo o ciclo de planejamento, desde a definição de objetivos comuns, estratégias de ação, definição de metas e recursos para alcança-las, assim como das formas de monitoramento e avaliação. Desta forma, cada setor poderá identificar e compreender melhor suas responsabilidades na agenda comum, priorizando em sua agenda específica essas ações. Ao mesmo tempo em que uma agenda setorial importante para a garantia de direitos pode ser mais valorizada e incentivada, se incluída no escopo de uma agenda intersetorial com um objetivo mais amplo.

Nesse sentido, a experiência em curso de diálogo entre a PNAN e a PNSAN, e entre o SUS e o SISAN, pode contribuir para a construção de outros diálogos entre políticas públicas para a promoção da saúde.

\section{Colaboradores}

KPS Alves trabalhou na concepção e na redação do artigo; e PC Jaime na concepção e na revisão crítica e aprovação da versão a ser publicada. 


\section{Referências}

1. Organização Mundial da Saúde. Declaração de Adelaide sobre a Saúde em Todas as Políticas: no caminho de uma governança compartilhada, em prol da saúde e do bem-estar. Relatório do encontro internacional sobre a Saúde em Todas as Políticas, Adelaide, 2010. [acessado 2014 ago 24] Disponível em: http://bvsms.saude.gov. br/bvs/publicacoes/declaracao_adelaide.pdf

2. Organização Mundial da Saúde. Declaração de Helsinki sobre a Saúde em Todas as Políticas. 8 Conferência Global sobre Promoção da Saúde, Helsinki, Finlândia, 2013. [acessado 2014 ago 24] Disponível em: http:// www.who.int/healthpromotion/conferences/8gchp/en/

3. Brasil. Ministério da Saúde (MS). Secretaria de Vigilância em Saúde. Política Nacional de Promoção da Saúde. Brasília: MS; 2006.

4. Vasconcelos FA. Combate à fome no Brasil: uma análise histórica de Vargas a Lula. Rev Nut 2005; 18(4):439-457.

5. Leão MM, Castro IRR. Políticas públicas de alimentação e nutrição In: Kac, G, Sichieri R, Gigante DP, organizadores. Epidemiologia Nutricional. Rio de Janeiro: Fiocruz, Atheneu; 2007. p. 519-541.

6. Arruda BKG, Arruda IKG. Marcos referenciais da trajetória das políticas de alimentação e nutrição no Brasil. Rev Bras Saude Mater Infant 2007; 7(3):319-326.

7. Frozi DS, Galeazzi MAM. Políticas Públicas de Alimentação no Brasil: uma revisão fundamentada nos conceitos de bem estar social e segurança alimentar e nutricional. Cad Debate 2004; XI:58-83.

8. Anjos LA, Burlandy L. Construção do conhecimento e formulação de políticas públicas no Brasil na área de segurança alimentar. Cien Saude Colet 2010; 15(1):1930.

9. Arruda BKG, Arruda IKG. Políticas de Alimentação e Nutrição no Brasil: breve enfoque dos delineamentos conceituais e propositivos. In: Taddei, JAAC, Lang, RMF, Silva, GL, Toloni, MHA, organizadores. Nutrição em Saúde Pública. Rio de Janeiro: Rubio; 2012. p. $397-$ 410.

10. Brasil. Lei no 11.346 de 15 de setembro de 2006. Cria o Sistema Nacional de Segurança Alimentar e Nutricional - SISAN com vistas a assegurar o direito humano à alimentação adequada e dá outras providências. Diário Oficial da União 2006; 18 set.

11. Brasil. Emenda Constitucional no 64 , de 4 de fevereiro de 2010. Altera o art. $6^{\circ}$ da Constituição Federal para introduzir a alimentação como direito social. Diário Oficial da União 2010; 4 fev.

12. Valente FLS, Burity V, Franceschini T, Carvalho MF Segurança Alimentar e Nutricional e o Direito Humano à Alimentação Adequada. In: ABRANDH. Curso de formação à distância em Direito Humano à Alimentação Adequada no contexto da Segurança Alimentar e Nutricional - Módulo 1. Brasília: ABRANDH; 2007.

13. Valente FLS, Burity V, Franceschini T. O DHAA, a SAN, a saúde e a promoção da alimentação saudável e adequada. In: ABRANDH. Curso de formação à distância em Direito Humano à Alimentação Adequada no contexto da Segurança Alimentar e Nutricional - Módulo 7. Brasília: ABRANDH; 2007.
14. Leão $\mathrm{MM}$, organizador. $O$ direito humano à alimentação adequada e o Sistema Nacional de Segurança Alimentar e Nutricional. Brasília: ABRANDH; 2013.

15. Buss PM, Pellegrini Filho A. A Saúde e seus Determinantes Sociais. Physis: Rev. Saúde Coletiva 2007; 17(1):77-93.

16. Pinheiro ARO. A alimentação saudável e a promoção da saúde no contexto da segurança alimentar e nutricional. RSD 2005; 29(70):125-139.

17. Burlandy L. Segurança Alimentar e Nutricional: intersetorialidade e ações de nutrição. Saúde em Revista 2004; 6(13):9-15.

18. Brasil. Ministério da Saúde (MS). Secretaria de Atenção à Saúde. Departamento de Atenção Básica. Política Nacional de Alimentação e Nutrição. 2. ed. rev. Brasília: MS; 2003.

19. Pinheiro ARO, Carvalho DBB. Estado e Mercado: adversários ou aliados no processo de implementação da Política Nacional de Alimentação e Nutrição? Elementos para um debate sobre medidas de regulamentação. Saúde e Sociedade 2008; 17(2):170-183.

20. Recine E, Vasconcellos AB. Políticas nacionais e o campo da Alimentação e Nutrição em Saúde Coletiva: cenário atual. Cien Saude Colet 2011; 16(1):73-79.

21. Brasil. Ministério da Saúde (MS). Secretaria de Atenção à Saúde. Departamento de Atenção Básica. Guia Alimentar para a população brasileira: promovendo a alimentação saudável. Brasília: MS; 2008.

22. World Health Organization (WHO). Global strategy on diet, physical activity and health. Food Nutr Bull 2004; 25(3):292-302.

23. Brasil. Ministério da Saúde (MS). Portaria no 4.279 de 30/12/2010. Estabelece diretrizes para a organização da Rede de Atenção à Saúde no âmbito do Sistema Único de Saúde (SUS). Diário Oficial da União 2010; 31 dez.

24. Brasil. Decreto no 7.272, de 25 de Agosto de 2010. Regulamenta a Lei no 11.346 , de 15 de setembro de 2006, que cria o Sistema Nacional de Segurança Alimentar e Nutricional - SISAN com vistas a assegurar o direito humano à alimentação adequada, institui a Política Nacional de Segurança Alimentar e Nutricional - PNSAN, estabelece os parâmetros para a elaboração do Plano Nacional de Segurança Alimentar e Nutricional, e dá outras providências. Diário Oficial da União 2010; 26 ago.

25. Brasil. Ministério da Saúde (MS). Secretaria de Atenção à Saúde. Departamento de Atenção Básica e Comissão Intersetorial de Alimentação e Nutrição do Conselho Nacional de Saúde. Documento-base de subsídio do Seminário Estadual de Alimentação e Nutrição no SUS. Brasília: MS; 2010.

26. Brasil. Ministério da Saúde (MS). Secretaria de Atenção à Saúde. Departamento de Atenção Básica. Política Nacional de Alimentação e Nutrição. Brasília: MS; 2012.

27. Câmara Interministerial de Segurança Alimentar e Nutricional (CAISAN). Plano Nacional de Segurança Alimentar e Nutricional: 2012/2015. Brasília: CAISAN; 2011. 
28. Brasil. Ministério da Saúde (MS). Secretaria de Vigilância em Saúde. Departamento de Análise de Situação de Saúde. Plano de Ações Estratégicas para o Enfrentamento das Doenças Crônicas Não Transmissíveis (DCNT) no Brasil 2011-2022. Brasília: MS; 2011.

29. Brasil. Ministério da Saúde (MS). Portaria no 424, DE 19/03/2013. Redefine as diretrizes para a organização da prevenção e do tratamento do sobrepeso e obesidade como linha de cuidado prioritária da Rede de Atenção à Saúde das Pessoas com Doenças Crônicas (republicada). Diário Oficial da União 2013; 28 maio.

30. Brasil. Ministério da Saúde (MS). Secretaria de Gestão Estratégica e Participativa. Departamento de Apoio à Gestão Participativa. Políticas de promoção da equidade. Brasília: MS; 2013.

31. Brasil. Ministério da Saúde (MS). Secretaria de Gestão Estratégica e Participativa. Departamento de Apoio à Gestão Participativa. Política Nacional de Educação Popular em Saúde. Brasília: MS; 2012.

32. Brasil. Presidência da República. Decreto No 6.272, de 23 de novembro de 2007. Dispõe sobre as competências, a composição e o funcionamento do Conselho Nacional de Segurança Alimentar e Nutricional CONSEA. Diário Oficial da União 2007; 26 nov.

33. Brasil. Presidência da República. Decreto No 6.273, de 23 de novembro de 2007. Cria, no âmbito do Sistema de Segurança Alimentar e Nutricional - SISAN, a Câmara Interministerial de Segurança Alimentar e Nutricional. Diário Oficial da União 2007; 26 nov.

Artigo apresentado em 18/06/2014

Aprovado em 11/08/2014

Versão final apresentada em 12/08/2014 